\title{
Is child sexual abuse declining in Canada? Results from nationally representative retrospective surveys
}

\section{Shields; L. Tonmyr, PhD; W. Hovdestad, PhD}

This article has been peer reviewed.

Tweet this article

\begin{abstract}
Introduction: Numerous data sources suggest a decline in child sexual abuse (CSA) in the United States since the early 1990s. Some evidence also indicates that an earlier period of higher CSA incidence began following World War II. This study examines prevalence estimates of sexual abuse reported retrospectively as having occurred in childhood (ChSA) in two nationally representative surveys of the Canadian population.
\end{abstract}

Methods: Data are from 13931 respondents aged 18 to 76 years from the 2004/2005 Canadian Gender, Alcohol, and Culture: An International Study (GENACIS), and from 22169 household residents aged 18 years or older who participated in the 2012 Canadian Community Health Survey-Mental Health (CCHS-MH). We present inter- and intrasurvey comparisons of ChSA prevalence specific to sex and age groups.

Results: Findings from both surveys suggest a decline in CSA since 1993, consistent with declines observed in the United States. Results also suggest that 1946 to 1992 was a period of higher risk of CSA, relative to the period before 1946. The evidence was more robust for women.

Conclusion: Evidence of a decline in CSA in Canada since the early 1990s is encouraging, given the long-term debilitating effects with which it is associated. Continued monitoring is critical. The long-term negative effects associated with CSA underscore the importance of continuing to move from lower risk to zero risk.

Keywords: child abuse, child maltreatment, childhood sexual abuse, prevalence, childhood person year, change over time, cohort

\section{Introduction}

The impact of child sexual abuse (CSA)associated with impairment throughout the lifespan-is unequivocal. ${ }^{1,2}$ Less clear are the magnitude of this problem and how the prevalence may have changed over time. Much of the information available about the incidence of CSA comes from data collected by child protection professionals. In the United States, data from child welfare agencies and professionals serving children in schools, hospitals and daycares reflect rising rates of CSA incidence from the mid-1970s to the early 1990s..$^{3-5}$ It has been suggested that the increases observed over the 1970s and 1980 s in data compiled by agencies reflected increased public awareness and changes in identifying and reporting CSA, rather than a true rise in incidence. ${ }^{3}$ Data from the United States' National Child Abuse and Neglect Data System indicate that over the years from 1992 to 2013, CSA declined by $64 \%$, similar to the decline in forcible rape apparent in FBI statistics. ${ }^{6}$ This decline is better supported by the evidence than the increase, as it does not stem from changes in administrative procedures. ${ }^{7}$ The decline is also consistent with improvements in other areas of child well-being in the United States during this

\section{Highlights}

- Data from two nationally representative surveys of the Canadian population suggest a decline in child sexual abuse (CSA) since 1993, consistent with declines observed in the United States.

- Results also suggest that 1946 to 1992 was a period of higher risk of $\mathrm{CSA}$, relative to the period before 1946.

- Evidence was more robust for women than men.

- Although retrospective, populationbased, methodologically consistent surveys can provide evidence of trends in CSA, a more comprehensive picture could be gleaned from contemporaneous information collected in surveys of children.

- The long-term negative effects associated with CSA highlight the importance of continuing to progress to the elimination of CSA; ongoing surveillance is required and complacency is not an option. 
deriving more accurate estimates of CSA. ${ }^{3}$ In this paper we use the term "childhood sexual abuse" (ChSA) to refer to sexual abuse during childhood reported in such surveys.

Data from numerous American victimization surveys provide evidence of declining rates of sexual abuse of children since the early 1990s that parallel trends observed from child welfare agency data. ${ }^{13}$ Other survey data suggest an increase following World War II. ${ }^{3,14-16}$ Some researchers, ${ }^{17,18}$ however, have concluded that legislation, social climate and differences in response rates and definitions of ChSA account for the apparent increase.

Two recent Canadian surveys representative of the general adult population included questions on ChSA: the 2012 Canadian Community Health SurveyMental Health (CCHS-MH) ${ }^{19}$ and the 2004/2005 Canadian Gender, Alcohol, and Culture: An International Study (GENACIS) . 20,21 Our analysis compares prevalence estimates of ChSA (by age group) between and within these two surveys. We assessed the consistency of Canadian data with the declines in CSA reported by Finkelhor et al., ${ }^{6}$ and with reported increases postWorld War II. More specifically, we examined Canadian survey data for any correspondence with the previous findings that ChSA prevalence was relatively lower before the end of World War II, relatively higher from after World War II to the early 1990s and then relatively lower after the early 1990s.

\section{Methods}

\section{Data sources}

We used data from the Canadian GENACIS to provide ChSA estimates for 2004/2005. The Canadian GENACIS is part of a large, international, multidisciplinary collaboration covering a range of topics related to gender, culture and alcohol-use problems. For the Canadian arm of GENACIS, a sample was selected using random-digit dialling to be representative of the Canadian household population aged 18 to 76 years living in the 10 provinces in 2004/2005. ${ }^{20}$ Interviews were conducted by telephone using computer-assisted telephone interviewing. The survey excluded households without a residential phone line, persons who did not speak English or French and the institutionalized population. ${ }^{21}$ The response rate was $52.8 \%$ (number of completions divided by estimated total number of eligible households), ${ }^{20}$ with a responding sample of 14063 (6009 men and 8054 women). This study excluded respondents who did not answer the ChSA questions (51 men and 81 women), reducing the sample size to 13931 (5958 men and 7973 women).

We used data from the CCHS-MH to provide ChSA prevalence estimates for 2012. Statistics Canada conducted the CCHS-MH using a multistage, stratified, clustered sampling design. ${ }^{19}$ The target population for the CCHS-MH was household residents aged 15 years or older living in the 10 Canadian provinces. Most of the interviews were conducted in person $(87 \%)$. The survey excluded people living on reserves and other Aboriginal settlements; full-time members of the Canadian Forces; and the institutionalized population, together representing about $3 \%$ of the target population. The response rate was $68.9 \%,{ }^{19}$ yielding a sample of 25113 individuals aged 15 years or older. CCHS-MH respondents were asked for permission to share their survey data with Statistics Canada's partners, including the Public Health Agency of Canada; most ( $n=23$ 709; $94 \%$ ) consented. This article is based on data from the "share" file. The ChSA questions were asked only of respondents aged 18 or older ( $\mathrm{n}=22486)$. Respondents who refused to answer (159 men and 158 women) were excluded from the study, resulting in a sample of 22169 (9910 men and 12259 women).

\section{Measures}

GENACIS respondents were classified as having experienced ChSA if they answered "yes" to either of the following questions:

- "Before you were 16 years old, did anyone in YOUR FAMILY try to make you do sexual things or watch sexual things when you did NOT want to?"

- "Before you were 16 years old, did anyone OTHER THAN A FAMILY MEMBER try to make you do sexual things or watch sexual things when you did NOT want to?"

CCHS-MH respondents were asked about "things that happened to you before you were 16 in your school, in your neighbourhood, or in your family":
- "How many times did an adult force you or attempt to force you into any unwanted sexual activity, by threatening you, holding you down or hurting you in some way?"

- "How many times did an adult touch you against your will in any sexual way? By this, I mean anything from unwanted touching or grabbing, to kissing or fondling."

CCHS-MH respondents were classified as having experienced ChSA if they answered "one or more times" to either question.

\section{Analyses}

We used two methods to examine changes in ChSA prevalence over time. First, we compared prevalence estimates of ChSA for groups in the same age range in GENACIS and CCHS-MH. Second, we compared ChSA prevalence for different age groups within the same survey. Comparison of prevalence estimates by age groups within retrospective surveys of adults is commonly used to examine generational differences in the extent of CSA. ${ }^{3,22}$ This approach reduces the possibility of methodological differences resulting in apparent differences in prevalence.

We proposed that prevalence would be relatively higher in people who spent their childhood (ages $0-15$ years) during the period 1946 to 1992 , and relatively lower in those who were aged 0 to 15 years during the period before the end of World War II (before 1946) or after the early 1990s (1993 and after). We used a modified version of the conventional personyears approach to create a "childhood person year" unit of analysis. Childhood person years (CPYs) are the individual units of time when the people in the study population were, by virtue of being aged 0 to 15 years, potentially exposed to CSA. We calculated the percentage of their childhood years that those in each age group spent in the higher- and lower-risk periods. Table 1 indicates the number and percentage of CPYs (i.e. at ages $0-15$ years) that those in each age group (at the time of the survey) spent in the three time periods. Figure 1 is a graphical depiction of how we calculated the percentages of the CPYs for what we proposed to be the higher-risk period.

Table 1 illustrates the CPY calculations. Consider the age group 18 to 24 years, 
TABLE 1

Number and percentage of childhood person years (ages 0-15) spent in risk periods for child sex abuse (1993 or after, from 1946-1992, and 1945 or before) among respondents to GENACIS and CCHS-MH, by age group at the time surveyed

\begin{tabular}{|c|c|c|c|c|c|c|c|c|c|c|c|c|c|c|c|}
\hline \multirow{3}{*}{$\begin{array}{c}\text { Age } \\
\text { group } \\
\text { in years }\end{array}$} & \multirow{3}{*}{$\begin{array}{c}\text { Total } \\
\text { CPYs } \\
\text { (ages } \\
0-15 \text { ) }\end{array}$} & \multicolumn{7}{|c|}{ GENACIS 2004} & \multicolumn{7}{|c|}{ CCHS-MH 2012} \\
\hline & & \multirow{2}{*}{$\begin{array}{l}\text { Year of } \\
\text { birth }\end{array}$} & \multicolumn{6}{|c|}{ Number and \% of CPYs (ages 0-15) spent } & \multirow{2}{*}{$\begin{array}{l}\text { Year of } \\
\text { birth }\end{array}$} & \multicolumn{6}{|c|}{ Number and \% of CPYs (ages 0-15) spent } \\
\hline & & & \multicolumn{2}{|c|}{1993 or after } & \multicolumn{2}{|c|}{ 1946-1992 } & \multicolumn{2}{|c|}{1945 or before } & & \multicolumn{2}{|c|}{1993 or after } & \multicolumn{2}{|c|}{ 1946-1992 } & \multicolumn{2}{|c|}{1945 or before } \\
\hline $18-24$ & 112 & 1980-1986 & 42 & 38 & 70 & 63 & 0 & 0 & 1988-1994 & 97 & 87 & 15 & 13 & 0 & 0 \\
\hline $25-34$ & 160 & 1970-1979 & 3 & 2 & 157 & 98 & 0 & 0 & 1978-1987 & 55 & 34 & 105 & 66 & 0 & 0 \\
\hline $50-64$ & 240 & 1940-1954 & 0 & 0 & 219 & 91 & 21 & 9 & 1948-1962 & 0 & 0 & 240 & 100 & 0 & 0 \\
\hline $65-76$ & 192 & 1928-1939 & 0 & 0 & 45 & 23 & 147 & 77 & 1936-1947 & 0 & 0 & 137 & 71 & 55 & 29 \\
\hline $\begin{array}{l}77 \text { or } \\
\text { older }^{b}\end{array}$ & 368 & & & & & & & & $\begin{array}{l}1935 \text { or } \\
\text { earlier }\end{array}$ & 0 & 0 & 15 & 4 & 353 & 96 \\
\hline
\end{tabular}

Abbreviations: CPYs, childhood person years; GENACIS, Gender, Alcohol, and Culture: An International Study; CCHS-MH, Canadian Community Health Survey-Mental Health.

Note: 2004 is used as the year for GENACIS since the majority of respondents (93\%) were interviewed in 2004.

${ }^{\mathrm{a}}$ Total CPYs $=(\#$ of birth years in cohort $) \times 16$.

${ }^{\mathrm{b}}$ Assumes the age cohort extends to age 99.

shown in the first line of Table 1 . These respondents had a total of 112 CPYs (the 16 years represented in the $0-15$ years of childhood $\times$ the 7 possible ages included in the 18-24 age group, assuming that the 7 ages are equally represented). For GENACIS respondents in this age group, $70 \mathrm{CPYs}$ were spent in the years 1946 to 1992, the higher-risk period. Those aged 18 years (born in 1986) spent 7 years (1986-1992); those aged 19, 8 years; those aged 20, 9 years; and so on until the final group, those aged 24, who spent 13 years in the higher-risk period; for a combined total of 70 childhood person years $(7+8+9+$ $10+11+12+13)$.

Therefore, the members of the youngest GENACIS age group spent 70 out of their 112 CPYs $(63 \%)$ in the time period we hypothesized to be higher-risk and the remainder (38\%) in the time period (1993 or after) we hypothesized to be lower-risk. In Figure 1, each shaded box represents a $\mathrm{CPY}$ spent in the higher-risk period. For GENACIS respondents aged 18 to 24 , there are a total of 70 shaded boxes (higherrisk) out of a total of 112 CPYs. The remaining 42 boxes that would fall to the right in the lower-risk period (1993 or after) are not shown in the figure. Those aged 35 to 49 years spent all 240 of their CPYs in the higher-risk period from 1946 to 1992 and respondents aged 25 to 34 and 50 to 64 years spent nearly all of their CPYs in this period ( $98 \%$ and $91 \%$, respectively).
Members of the oldest GENACIS age group (65-76 years) lived the majority of their CPYs before the end of World War II $(147 / 192=77 \%)$, a period we hypothesized to be lower-risk. Note that in Figure 1, only 45 shaded boxes appear in the higher-risk period for this age group; the remaining 147 CPYs for this group would fall to the left in the lower-risk period before 1946. These CPYs were included in the analyses (in the lower-risk period) but are not shown in the figure.

Because CCHS-MH respondents were surveyed eight years later than GENACIS respondents, people who were then in the age groups used to classify GENACIS respondents would have spent different proportions of their childhood years in times of higher or lower risk. The four intermediate CCHS-MH age groups (25-34, $35-49,50-65$, and $65-76$ years) spent the majority of their CPYs in what we term the higher-risk period. Those aged 35 to 49 and 50 to 64 years spent all of their CPYs in this time period. Among those in the youngest (18-24 years) and oldest (77 years or older) age groups, the majority of CPYs were spent in what we have been calling the lower-risk periods (those in the youngest group spent $87 \%$ of their CPYs in the period 1993 or after, and those in the oldest group, $96 \%$ in the period before the end of World War II).
When comparing ChSA prevalence estimates between GENACIS and CCHS-MH, we assumed that the ChSA construct is the same in both surveys. Estimates may vary due to methodological differences between the surveys and/or changes over time in participants' willingness to disclose ChSA. To assess the potential for differences in methodology affecting the estimates, we compared estimates by matching cohorts with the same year-ofbirth groupings in the two surveys. Since there was an eight-year time lag between the two surveys, we would expect, for example, that prevalence of ChSA would be statistically similar between GENACIS respondents aged 18 to 24 and CCHS-MH respondents aged 26 to 32 (all born from 1980-1986), barring differences due to these other factors.

We conducted our analyses using SAS Enterprise Guide 5.1 (SAS Institute Inc; Cary, North Carolina, USA). All estimates are based on weighted data. Tests for significant differences between estimates from GENACIS and CCHS-MH were based on pooled $t$ tests. For CCHS-MH estimates, variance and $95 \%$ confidence intervals (CIs) were calculated using the bootstrap technique to account for the complex survey design of the CCHS-MH. ${ }^{19}$ For GENACIS estimates, variance and 95\% CIs were calculated assuming simple random sampling. 


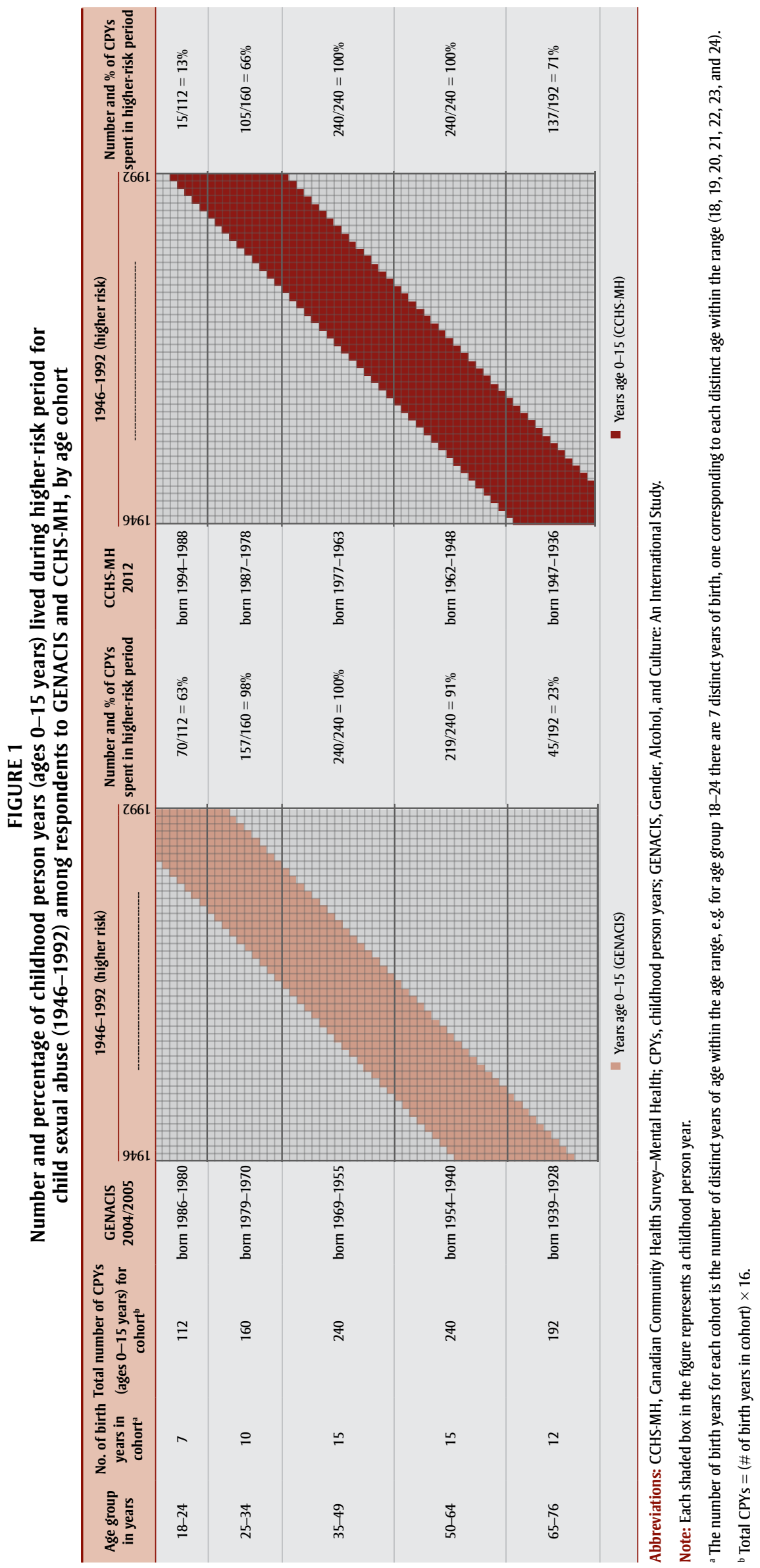

\section{Results}

ChSA prevalence estimates from GENACIS and CCHS-MH were similar for the population aged 18 to 76 years; i.e. $9.8 \%$ based on 2004/2005 GENACIS data, and 10.5\% based on 2012 CCHS-MH data (Table 2). However, differences by sex and age group emerged between the two time periods.

Among women in the youngest age group (18-24 years), prevalence declined from $10.0 \%$ in $2004 / 2005$ to $6.4 \%$ in 2012 . Among women aged 25 to 34 years, prevalence was $15.0 \%$ in $2004 / 2005$ and $12.1 \%$ in 2012, but the decline only approached statistical significance ( $p=.08$ ). Women in these two age groups at the time of the CCHS-MH would have spent a greater percentage of their childhood years in the period 1993 or after (lower-risk), compared with women in these age groups at the time of GENACIS.

Conversely, among women in the two oldest groups, prevalence increased between the two time periods; from $14.2 \%$ to $19.8 \%$ among those aged 50 to 64 years, and from $6.2 \%$ to $13.2 \%$ among those aged 65 to 76 . Women in these age groups at the time of the CCHS-MH spent a greater percentage of childhood in the years 1946 to 1992 (higher-risk) compared with women in these age groups at the time of GENACIS.

Among women, a U-shaped association was observed between age and ChSA. In $2004 / 2005$, prevalence of ChSA was highest for those aged 35 to 49 years (who spent all childhood years in the higherrisk period 1946-1992). In 2012, it was highest for those aged 35 to 49 and 50 to 64 years (who also spent all childhood years in this higher-risk period). In both time periods, the lowest prevalence estimates were observed for those in the youngest and oldest age groups. Women in the youngest group spent a greater proportion of childhood years in the period 1993 or after, while those in the oldest age group spent more childhood years in the period before the end of World War II (both periods of lower risk).

Among men, the only significant difference observed between the surveys was for those aged 65 to 76 years, for whom prevalence increased from $6.3 \%$ in $2004 / 2005$ to $10.0 \%$ in 2012 . Men aged 65 to 76 in 2012 spent $71 \%$ of their childhood 
TABLE 2

Prevalence of childhood sexual abuse by sex and age group at time surveyed, household population aged 18 or older, Canada, 2004/2005 and 2012

\begin{tabular}{|c|c|c|c|c|c|c|c|c|c|}
\hline & \multicolumn{4}{|c|}{ 2004/2005 (GENACIS) } & \multicolumn{4}{|c|}{2012 (CCHS-MH) } & \multirow{2}{*}{$\begin{array}{c}2012 \text { vs. } \\
2004 / 2005\end{array}$} \\
\hline & Year of birth & $\%$ & $(95 \% \mathrm{CI})$ & & Year of birth & $\%$ & $(95 \% \mathrm{Cl})$ & & \\
\hline Total (ages 18-76) & 1928-1986 & 9.8 & $(9.2-10.3)$ & & 1936-1994 & 10.5 & $(9.9-11.2)$ & & \\
\hline Male (ref) & 1928-1986 & 5.4 & $(4.8-6.0)$ & & 1936-1994 & 5.9 & $(5.2-6.7)$ & & \\
\hline Female & 1928-1986 & 14.0 & $(13.2-14.9)$ & a & 1936-1994 & 15.0 & $(14.0-16.1)$ & a & \\
\hline $18-24$ & 1980-1986 & 6.0 & $(4.6-7.4)$ & a & 1988-1994 & 4.3 & $(3.3-5.4)$ & a & \\
\hline $25-34$ & 1970-1979 & 9.5 & (8.3-10.8) & a & 1978-1987 & 7.6 & $(6.2-9.1)$ & a & \\
\hline $35-49$ (ref) & 1955-1969 & 12.0 & $(11.0-13.0)$ & & 1963-1977 & 11.5 & $(10.1-12.9)$ & & \\
\hline $50-64$ & 1940-1954 & 10.4 & $(9.3-11.4)$ & a & 1948-1962 & 13.5 & $(12.1-14.8)$ & a & b \\
\hline \multicolumn{10}{|c|}{ Males age group (in years) } \\
\hline $18-24$ & 1980-1986 & 2.2 & $(1.0-3.5)$ & a & 1988-1994 & 2.3 & $(1.1-3.4)$ & a & \\
\hline $25-34$ & 1970-1979 & 4.3 & $(2.9-5.6)$ & a & 1978-1987 & 3.4 & $(2.1-4.7)$ & a & \\
\hline $35-49$ (ref) & 1955-1969 & 6.4 & $(5.2-7.5)$ & & 1963-1977 & 6.0 & $(4.5-7.5)$ & & \\
\hline $50-64$ & 1940-1954 & 6.4 & $(5.1-7.8)$ & & 1948-1962 & 7.3 & $(5.6-9.0)$ & & \\
\hline $65-76$ & 1928-1939 & 6.3 & $(4.2-8.4)$ & & 1936-1947 & 10.0 & (7.5-12.6) & a & b \\
\hline 77 or older & & & & & 1935 or earlier & 4.7 & $(2.7-6.7)$ & & \\
\hline \multicolumn{10}{|c|}{ Females age group (in years) } \\
\hline
\end{tabular}

Sources: GENACIS Canadian Survey 2004/2005; Statistics Canada, CCHS-MH, 2012 (share file).

Abbreviations: CCHS-MH, Canadian Community Health Survey-Mental Health; Cl, confidence interval; GENACIS, Gender, Alcohol, and Culture: An International Study; ref, reference group. Note: The GENACIS estimates include 239 records with a missing value for age (excluded from estimates by age group).

a Significantly different from reference.

b Significantly different from 2004/2005.

years in the higher-risk period from 1946 to 1992 , compared with $23 \%$ for men in this age group at the time of GENACIS.

Among men, the prevalence of ChSA in 2012 was highest $(10.0 \%)$ among those aged 65 to 76 years and was lower for the two youngest age groups, who spent more of their childhood years in the lower-risk period of 1993 or after.
Comparisons based on matched year-ofbirth cohorts between the two surveys (Table 3) revealed statistically similar estimates for most of the matched year-ofbirth groupings (which would be expected, barring methodological differences between the two surveys). However, significant differences were observed for men born from 1940 to 1954 and women born 1940 to 1954 and 1928 to 1939: prevalence was higher among CCHS-MH respondents.

\section{Discussion}

This study of ChSA prevalence estimates from two large, representative surveys of the Canadian adult population supports our hypothesis that 1993 and after was a period of lower risk for CSA, 1946 to 1992 was a period of higher risk, and the time before 1946 was a period of lower risk. The evidence was substantial for women. The lower prevalence estimates for younger 
TABLE 3

Prevalence of childhood sexual abuse by sex and matched date-of-birth cohorts, household population aged 18 to 76 years, Canada, 2004/2005 and 2012

\begin{tabular}{|c|c|c|c|c|c|c|c|}
\hline \multirow[b]{2}{*}{$\begin{array}{l}\text { Year of birth } \\
\text { cohort }\end{array}$} & \multicolumn{3}{|c|}{ 2004/2005 (GENACIS) } & \multicolumn{3}{|c|}{2012 (CCHS-MH) } & \multirow[b]{2}{*}{$\begin{array}{c}2012 \text { vs. } \\
2004 / 2005\end{array}$} \\
\hline & $\begin{array}{l}\text { Age group at time of } \\
\text { GENACIS }\end{array}$ & $\begin{array}{l}\text { \% reporting } \\
\text { ChSA }\end{array}$ & $(95 \% \mathrm{Cl})$ & $\begin{array}{l}\text { Age group at time of } \\
\text { CCHS }\end{array}$ & $\begin{array}{l}\text { \% reporting } \\
\text { ChSA }\end{array}$ & $(95 \% \mathrm{Cl})$ & \\
\hline 1980-1986 & $18-24$ & 2.2 & $(1.0-3.5)$ & $26-32$ & 2.5 & $(1.1-3.8)$ & \\
\hline 1955-1969 & $35-49$ & 6.4 & $(5.2-7.5)$ & $43-57$ & 6.9 & $(5.3-8.5)$ & \\
\hline 1940-1954 & $50-64$ & 6.4 & $(5.1-7.8)$ & $58-72$ & 9.1 & (7.0-11.1) & a \\
\hline \multirow[t]{2}{*}{ 1928-1939 } & $65-76$ & 6.3 & $(4.2-8.4)$ & $73-84$ & 5.1 & $(3.4-6.9)$ & \\
\hline & $\begin{array}{l}\text { Females age group } \\
\text { (in years) }\end{array}$ & & & $\begin{array}{l}\text { Females age group } \\
\text { (in years) }\end{array}$ & & & \\
\hline 1955-1969 & $35-49$ & 17.6 & $(16.0-19.1)$ & $43-57$ & 18.5 & $(16.1-20.9)$ & \\
\hline 1940-1954 & $50-64$ & 14.2 & $(12.6-15.8)$ & $58-72$ & 17.3 & (15.4-19.1) & a \\
\hline 1928-1939 & $65-76$ & 6.2 & $(4.5-7.9)$ & 73-84 & 10.0 & $(7.5-12.4)$ & a \\
\hline
\end{tabular}

Sources: GENACIS, Canadian Survey 2004/2005; Statistics Canada, CCHS-MH, 2012 (share file).

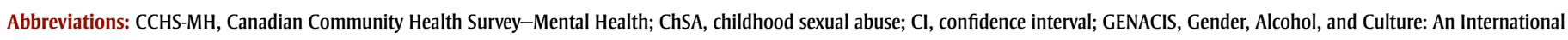
Study.

${ }^{\text {a }}$ Significantly different from 2004/2005.

men in the CCHS-MH provide weak evidence of a possible slight decline since the early 1990s. However, further surveillance is required to substantiate a decline in CSA among Canadian males.

\section{Evidence and reasons for the decline}

Our findings regarding the decline (or lower risk) of CSA since the early 1990s are consistent with findings from Finkelhor et al., based on many American data sources, ${ }^{6,13}$ and with findings from a study based on a nationally representative survey of Australians. ${ }^{22}$ Studies of data collected from students also provide evidence of a decline during this time period. For example, in Aotearoa, New Zealand, reports of CSA declined between 2001 and 2007, with a greater decrease being reported for males. ${ }^{23}$ In British Columbia, Canada, a decline was observed between 1992 and 2003. ${ }^{24}$ Data from child welfare authorities captured in the Canadian Incidence Study of Reported Child Abuse and Neglect (CIS) also suggest that declines have occurred..$^{25,26}$ Although concerns have been raised about the national representativeness of estimates based on CIS weighted data, ${ }^{27}$ estimates indicate a decline in the number of substantiated sexual abuse investigations per 1000 children aged 0 to 15 , from 86 in 1998 to 43 in $2008 .^{25,26}$ Likewise, policereported sexual offences in Canada peaked in 1993 and then declined throughout the late 1990 s and into the 2000 s. $^{12}$

Finkelhor and Jones ${ }^{9}$ reviewed numerous potential explanations for the decline in CSA observed in the United States and concluded that multiple factors likely contributed. They proposed that economic prosperity, increases in policing and other agents of social intervention such as social and child protection workers, and psychiatric pharmacotherapy were the most plausible explanations. They also suggested that the increasing availability of effective contraception in the years leading up to and during the period of the CSA declines resulted in fewer unplanned births, and a greater likelihood that children would be raised in environments with adequate social and economic resources to ensure their protection. Changing norms and practices may have also played a role; rising levels of education may have heightened awareness of CSA and the importance of providing safe environments for children.

Some of the explanations for CSA declines in the United States may not apply to the Canadian context. A study comparing reasons for the general drop in crime observed in the 1990s in Canada and the United States suggested somewhat different explanations for the two countries. ${ }^{28}$ In Canada, in contrast to the United States, policing and incarceration rates changed little or not at all during the decline; rather, changes in demographics, employment rates and collective values were more likely explanations. ${ }^{28}$ Canadian data sources support other possible explanations proposed by Finkelhor and Jones, ${ }^{9}$ including declines in teen pregnancy, ${ }^{29}$ increases in educational levels ${ }^{30}$ and decreases in unemployment. ${ }^{28}$ The use of antidepressants also increased in Canada from the early 1990s. ${ }^{31}$ Parents with mental disorders who can obtain treatment may be better able to attend to and protect their children than parents who lack necessary treatment. ${ }^{9}$ 
Finally, CSA prevention programs within schools and campaigns to raise awareness of the issue may have had a positive effect. $^{32,33}$

\section{Evidence and reasons for increased risk}

Evidence to substantiate an increase in CSA in the post-World War II period is less clear. The paucity of surveys conducted in these years and inconsistencies in methodologies of the surveys that were conducted reduce the usefulness of findings related to this time period. Methodological inconsistencies preclude direct comparisons of GENACIS and CCHS-MH with a 1983 Canadian survey. ${ }^{34}$ However, results from this survey support our hypothesis of a higher risk of CSA in the post-World War II period. Although this survey provides limited information specific to age groups, one report states that prevalence of ChSA among those under 25 years was twice that in those aged 44 or older. ${ }^{14}$ Those under 25 would have spent all of their childhood years in the period we term "higher-risk" (1946-1992) while those 44 or older would have spent the majority of their childhood years in the “lower-risk" period prior to 1946. Results from other surveys also suggest an increase of CSA during the post-World War II era. ${ }^{3,15,16}$

Changes in family structure post-World War II may have contributed to the increase. ${ }^{14,35}$ Before World War II, divorce in Canada was close to non-existent. After World War II, divorce became more common, and rates peaked in the late 1980s after amendments were made to the Divorce Act. ${ }^{35}$ Since the early 1990s, divorce rates have been fairly stable. Several studies of CSA that occurred during the 1970s and 1980s suggest that children living with a single parent or a stepparent had a greater risk of CSA than those living with two biological parents, ${ }^{36-38}$ possibly accounting for the increase in CSA in the post-World War II era. However, the characteristics of stepand single-parent families of the 1990s and later may differ from these earlier families. The other factors cited previously likely account for the decline in CSA during the period of stable divorce rates (i.e. since the early 1990s).

\section{Strengths and limitations}

Our study uses a novel approach (that of "childhood person years") to illustrate evidence of trends in CSA in Canada using two large representative surveys of the Canadian population. The epidemiology of CSA is difficult to measure. The childhood-person-years approach may assist future work in providing evidence of CSA trends based on prevalence of ChSA among adults and adolescents from retrospective surveys.

Although the use of retrospective surveys to examine trends in CSA addresses some of the problems related to the underreporting of CSA inherent in agency data, survey data are subject to some limitations. While false positive reports of ChSA in retrospective surveys are rare, ${ }^{39}$ the rate of false negatives is substantial-ranging from $40 \%$ to $50 \% .40-42$ There is evidence that underreporting is more common among males. ${ }^{42,43}$ Although the specific items used to measure ChSA in the CCHS-MH and GENACIS have not been validated, similar items were used in the Canadian General Social Survey ${ }^{12}$ and the items are behaviour-specific and thus likely to have higher validity and reliability than broad and subjectively defined questions. ${ }^{39}$ Finally, both surveys exclude people residing in institutions and those without fixed addresses, populations in which CSA would likely be more prevalent.

Discrepancies in estimates from different survey sources may arise from methodological inconsistencies rather than true differences in prevalence. The items used to measure ChSA differed between GENACIS and the CCHS-MH, as did response rates and modes of administration. As well, CCHS-MH respondents were asked four items about other forms of childhood maltreatment before the two ChSA items; GENACIS respondents experienced no such priming. Despite these differences, comparisons by matched year-of-birth cohorts showed similar prevalence estimates for the three youngest age cohorts, suggesting that the two surveys were measuring a similar construct. However, the extent to which response rates, particularly the low response rate to GENACIS, affect prevalence estimates of ChSA is unknown.

While comparing prevalence by age group within surveys avoids some of the shortcomings associated with methodological inconsistencies between surveys, some limitations remain. The likelihood of reporting varies by age, in that people may not disclose their CSA experiences until many years have passed. ${ }^{43}$ However, lower prevalence among older respondents may result partly from premature mortality among people who have experienced CSA. ${ }^{44,45}$ As the length of time since the CSA occurred increases, so too will downward biases on prevalence estimates due to premature mortality; people who have died cannot be in the sample and report their ChSA. Therefore, evidence of an increase between the pre- and postWorld War II periods is likely to be erroneously exaggerated, and evidence of a decline since 1993 may be somewhat understated.

Secular trends may also affect the likelihood of acknowledging ChSA. Comparisons of matched year-of-birth cohorts revealed higher prevalence in the CCHS-MH than in GENACIS for women born 1928 to 1954 and men born 1940 to 1954 . These findings suggest an increased likelihood for these groups to disclose ChSA, perhaps as an effect of media coverage of high-profile cases $^{46}$ or other media attention to the issue of sexual violence. Note that frequent or intense media attention may also result in the public perception that CSA is increasing, independent of actual trends in its incidence. Although the likelihood of disclosing may increase with age, in general, the lower prevalence among older respondents may relate to a persistent reluctance to disclose among these individuals who were born in an era when having been sexually victimized may have been more stigmatizing.

Given these limitations, the novelty of our CPY method and the paucity of similar prior analyses using Canadian data, we encourage replication of our study in Canada and elsewhere to provide additional evidence for a real decline in CSA.

\section{Conclusion}

Evidence for a decline in CSA in Canada since the early 1990s, consistent with a decline in the United States during the same timeframe, is an encouraging finding. However, in 2012, among those aged 18 to $24,6 \%$ of women and $2 \%$ of men reported ChSA-figures that most likely understate the true magnitude of occurrence. Continued monitoring of the prevalence of ChSA is critical. Retrospective population-based surveys, using items with sound psychometric properties and 
consistent methodologies are effective means of providing evidence of trends in CSA. A more complete picture, however, could be gleaned from contemporaneous data sources such as information collected in surveys of students. High quality data are essential for evaluating intervention programs aimed at eradicating sexual abuse of Canadian children. The longterm negative effects associated with CSA underscore the importance of continuing to move from lower risk to zero risk; complacency is not an option.

\section{Acknowledgements}

Data collected as part of the international project Gender, Alcohol and Culture: An International Study (GENACIS), were provided by the Institute for Social Research (ISR), York University. The survey was completed on behalf of the Centre for Addiction and Mental Health (CAMH). Funding for the Canadian arm of GENACIS was provided through an operations grant from the Canadian Institutes of Health Research (CIHR). Neither CAMH, ISR nor CIHR are responsible for the analyses and interpretations presented here.

The authors gratefully acknowledge Kathryn Wilkins' assistance with developing the clearest possible expression of the complex constructs used in these analyses.

\section{References}

1. Gilbert R, Widom CS, Browne K, Fergusson D, Webb E, Janson S. Burden and consequences of child maltreatment in high-income countries. Lancet. 2009;373(9657):68-81. doi: 10.1016/S0140-6736(08)61706-7.

2. Maniglio R. The impact of child sexual abuse on health: a systematic review of reviews. Clin Psychol Rev. 2009;29(7):647-57. doi: 10.1016/j.cpr .2009.08.003.

3. Finkelhor D. Current information on the scope and nature of child sexual abuse. Future Child. 1994;4(2):31-53. doi: $10.2307 / 1602522$.

4. Lindsey D. Mandated reporting and child abuse fatalities: requirements for a system to protect children. Soc Work Res. 1994;18(1):41-54.

5. Sedlack AJ, Broadhurst DD. Third National Incidence Study of Child Abuse and Neglect. Washington (DC): U.S. Department of Health and Human Services; 1996.
6. Finkelhor D, Saito K, Jones L. Updated trends in child maltreatment, 2013. Durham (NH): Crimes against Children Research Center; 2015.

7. Almeida J, Cohen AP, Subramanian SV, Molnar BE. Are increased worker caseloads in state child protective service agencies a potential explanation for the decline in child sexual abuse? A multilevel analysis. Child Abuse Negl. 2008;32(3):367-75. doi: 10.1016/j .chiabu.2007.08.003.

8. Finkelhor D, Jones LM. Explanations for the decline in child sexual abuse cases. Juvenile Justice Bulletin. Washington (DC): Office of Juvenile Justice and Delinquency Prevention; 2004.

9. Finkelhor D, Jones LM. Why have child maltreatment and child victimization declined? J Soc Issues. 2006; 62(4):685-716. doi: 10.1111/j.1540-4560 .2006.00483.x.

10. Land KC, Lamb VL, Kahler Mustillo S. Child and youth well-being in the United States, 1975-1998: some findings from a new index. Soc Indic Res. 2001;56(3):241-320. doi: 10.1023 /A:1012485315266.

11. Afifi TO, MacMillan HL, Taillieu T, et al. Relationship between child abuse exposure and reported contact with child protection organizations: results from the Canadian Community Health Survey. Child Abuse Negl. 2015;46: 198-206. doi: 10.1016/j.chiabu.2015.05 .001 .

12. Brennan S, Taylor-Butts A. Sexual assault in Canada 2004 and 2007. Canadian Centre for Justice Statistics Profile Series. Ottawa (ON): Statistics Canada; 2008. [Statistics Canada, Catalogue No.: 85F0033M].

13. Finkelhor D, Jones LM. Have sexual abuse and physical abuse declined since the 1990s? Durham (NH): Crimes against Children Research Center; 2012.

14. Bagley C. Child sexual abuse in Canada: further analysis of the 1983 national survey. Ottawa (ON): Health and Welfare Canada; 1988.

15. Leventhal JM. Have there been changes in the epidemiology of sexual abuse of children during the 20th century? Pediatrics. 1988;82(5): 766-73.
16. Wyatt GE, Loeb TB, Solis B, Carmona JV, Romero, G. The prevalence and circumstances of child sexual abuse: changes across a decade. Child Abuse Negl. 1999;23(1):45-60. doi: 10.1016 /S0145-2134(98)00110-0.

17. Feldman W, Feldman E, Goodman JT, et al. Is childhood sexual abuse really increasing in prevalence? An analysis of the evidence. Pediatrics. 1991;88(1): 29-33. doi: 10.1016/s0890-8567(10) 80016-4.

18. Gorey KM, Leslie DR. The prevalence of child sexual abuse: integrative review adjustment for potential response and measurement biases. Child Abuse Negl. 1997;21(4):391-8. doi: 10.1016/s0145-2134(96)00180-9.

19. Statistics Canada. Canadian Community Health Survey (CCHS) - Mental Health User Guide. Statistics Canada; 2013.

20. Graham K, Bernards S. Canada: alcohol and partner physical aggression in the 10 provinces. In: Graham K, Bernards S, Munné M, Wilsnack S, eds. Unhappy Hours: Alcohol and Physical Partner Aggression in the Americas. Washington (DC): Pan American Health Organization; 2008: 90-108.

21. Graham K, Massak A. Alcohol consumption and the use of antidepressants. CMAJ. 2007;176(5):6337. doi: $10.1503 / \mathrm{cmaj} .060446$.

22. Dunne MP, Purdie DM, Cook MD, Boyle FM, Najman JM. Is child sexual abuse declining? Evidence from a population-based survey of men and women in Australia. Child Abuse Negl. 2003;27(2):141-52. doi: 10.1016 /s0145-2134(02)00539-2.

23. Denny SJ, Grant S, Utter J, et al. Health and well-being of young people who attend secondary school in Aotearoa, New Zealand: what has changed from 2001 to 2007? J Paediatr Child Health. 2011;47(4):191-7. doi: 10.1111/j.1440-1754.2010.01945.x.

24. Tonkin RS. British Columbia Youth Health Trends: A Retrospective, 19922003. Vancouver, BC: McCreary Centre Society; 2005. 
25. Public Health Agency of Canada. Canadian Incidence Study of Reported Child Abuse and Neglect - 2008: major findings. Ottawa (ON): Public Health Agency of Canada; 2010.

26. Trocmé N, MacLaurin B, Fallon B, et al. Canadian Incidence Study of Reported Child Abuse and Neglect: Final Report. Ottawa (ON): Minister of Public Works and Government Services Canada; 2001.

27. Dumais J. Comparison and assessment of CIS 1998, 2003 and 2008 designs. Ottawa $(\mathrm{ON})$ : Statistics Canada; 2010. Unpublished report.

28. Ouimet M. Explaining the American and Canadian crime "drop" in the 1990s. Can J Criminol. 2002;44(1): 33-50.

29. McKay A. Trends in Canadian national and provincial/territorial teen pregnancy rates 2001-2010. Can J Hum Sex. 2013;21(3-4):161-75.

30. Statistics Canada, Council of Ministers of Education, Canada. Education indicators in Canada: an international perspective. Ottawa (ON); 2014. [Statistics Canada, Catalogue No.: 81-604-X].

31. Hemels ME, Koren G, Einarson TR. Increased use of antidepressants in Canada: 1981-2000. Ann Pharmacother. 2002;36(9):1375-9. doi: 10.1345/aph $.1 \mathrm{a} 331$.

32. MacMillan HL, Wathen CN, Barlow J, Fergusson DM, Leventhal JM, Taussig HN. Interventions to prevent child maltreatment and associated impairment. Lancet. 2009;373(9659):250-66. doi: 10.1016/S0140-6736(08)61708-0.

33. Walsh K, Zwi K, Woolfenden S, Shlonsky A. School-based education programmes for the prevention of child sexual abuse. Cochrane Database Syst Rev. 2015;4:CD004380. doi: 10.1002 /14651858.CD004380.pub3.

34. Badgley RF, Allard HA, McCormick $\mathrm{N}$, et al. Sexual offenses against children. Ottawa (ON): Department of Supply and Services; 1984.

35. Milan A. Marital status: overview, 2011. Report on the demographic situation in Canada. Ottawa (ON): Statistics Canada; 2013 [Statistics Canada, Catalogue No.: 91-209-X].
36. Daly M, Wilson M. Child abuse and other risks of not living with both parents. Ethol Sociobiol. 1985;6(4): 197-210.

37. Fleming J, Mullen P, Bammer G. A study of potential risk factors for sexual abuse in childhood. Child Abuse Negl. 1997;21(1):49-58. doi: 10.1016/s0145-2134(96)00126-3.

38. Giles-Sims, J. Current knowledge about child abuse in stepfamilies. Marriage Fam Rev. 1997;26:215-230.

39. Hardt J, Rutter M. Validity of adult retrospective reports of adverse childhood experiences: review of the evidence. J Child Psychol Psychiatry. 2004;45(2):260-73. doi: 10.1111/j.1469 $-7610.2004 .00218 . x$.

40. Fergusson DM, Horwood LJ, Woodward LJ. The stability of child abuse reports: a longitudinal study of the reporting behaviour of young adults. Psychol Med. 2000;30(3):529-44. doi: 10.1017 /s0033291799002111.

41. Scott KM, Smith DR, Ellis PM. Prospectively ascertained child maltreatment and its association with DSM-IV mental disorders in young adults. Arch Gen Psychiatry. 2010;67(7): 712-9.

42. Widom CS, Morris S. Accuracy of adult recollections of childhood victimization: Part 2. Childhood sexual abuse. Psychol Assess. 1997;9(1):3446. doi: 10.1037/1040-3590.9.1.34.

43. Hébert $M$, Tourigny $M$, Cyr $M$, McDuff P, Joly J. Prevalence of childhood sexual abuse and timing of disclosure in a representative sample of adults from Quebec. Can J Psychiatry. 2009;54(9):631-6. doi: 10.1177 /070674370905400908.

44. Brown DW, Anda RF, Tiemeier H, et al. Adverse childhood experiences and the risk of premature mortality. Am J Prev Med. 2009;37(5):389-96. doi: 10.1016/j.amepre.2009.06.021.

45. Felitti VJ, Anda RF, Nordenberg D, et al. Relationship of childhood abuse and household dysfunction to many of the leading causes of death in adults. The Adverse Childhood Experiences (ACE) Study. Am J Prev Med. 1998; 14(4):245-58. doi: 10.1016/s0749-3797 (98)00017-8.
46. Weatherred JL. Child sexual abuse and the media: a literature review. J Child Sex Abus. 2015;24(1):16-34. doi: $10.1080 / 10538712.2015 .976302$. 